\title{
Accidental Catheterization of Left Partial Anomalous Pulmonary Venous Connection (PAPVC): A Rare Instance of Central Venous Access Malpositioning
}

\author{
Shibojit Talukder ${ }^{1 *}$, Srinath $\mathrm{SR}^{1}$, Arunanshu Behera ${ }^{1}$ and Deeban Ganeshan ${ }^{1}$
}

${ }^{1}$ Department of General Surgery, PGIMER, Chandigarh, India

Received: 20 May, 2017 ; Accepted: 14 June, 2017; Published: 23 June, 2017

*Corresponding author: Shibojit Talukder, MS, Senior Resident, Department Of General Surgery, PGIMER, Chandigarh, India, Mobile no: +919914017546; E-mail: Shibojitsplace@Gmail.Com

\begin{abstract}
Central venous catheter (CVC) is one of the most commonly placed invasive devices in critical care setup. Its placement can be associated with many technical complications like bleeding, pneumothorax, arterial puncture and infection. Furthermore technical and anatomical variations, unfavorable body habitus can lead to malpositioning of CVC within venous system even when they are placed under radiological guidance. If not addressed timely, malpositioning can be associated with poor catheter functioning and serious complications like vessel erosion, bleeding, thrombosis. Presence of congenital venous anomalies can be a rare cause of CVC malpositioning. We report a case of young female in whom CVC placed through left internal jugular venous (IJV) route accidentally entered into a left upper pulmonary vein through partial anomalous pulmonary venous connection (PAPVC) with the left brachiocephalic vein. Post insertion chest radiograph showed unusual curvilinear course of the left IJV access towards left pulmonary hilum. The diagnosis was clinched after contrast enhanced computed tomography delineated the previously undiagnosed venous anomaly in the patient. There is no report of CVC malposition into the pulmonary vein in medical literature making it unique and first of its kind. Malpositioned CVC can thus lead to revelation of asymptomatic congenital vascular anomalies in a completely unrelated clinical setting.
\end{abstract}

Keywords: Central Venous Catheter (CVC); Partial Anomalous Pulmonary Venous Connection (PAPVC); Catheter Malpositioning

\section{Abbreviations}

CVC: Central Venous Access; PAPVC: Partial Anomalous Pulmonary Venous Connection ; IJV- Internal Jugular Vein

\section{Introduction}

Central venous catheter (CVC) placement is an important invasive intervention which is commonly undertaken in critically ill patients with the intent of central venous pressure measurement, drug, nutritional and renal replacement therapy, cardiac pacing. It is not uncommon to encounter complications during its placement. It is recommended that CVC should be placed under ultrasound guidance. Post placement check radiograph is mandatory to confirm proper tip positioning and rule out puncture related complications. Finding poorly functioning CVC or abnormal course of the catheter on radiograph should prompt evaluation for the reason. Catheter malpositioning if found should be addressed in order to ensure optimal use and prevent serious complications arising thereof. Sometimes asymptomatic congenital venous anomalies may be brought into clinical light by malpositioning incidence. We report a case of asymptomatic left sided partial anomalous pulmonary venous connection (PAPVC) in a young female which was accidentally catheterized during a left sided CVC placement.

\section{Case History}

A 26 year female suffering from end stage renal disease on maintenance hemodialysis developed iatrogenic left superficial femoral artery pseudoaneurysm following placement of temporary trans-femoral dialysis access. She presented to our center with bleeding from the ruptured pseudoaneurysm. She was in shock and required immediate transfer to operating room. A left sided internal jugular venous access (IJVA) was established during surgery due to lack of adequate peripheral vascular access for resuscitation. Check radiographs in postoperative recovery room showed that the IJVA was directed towards the left pulmonary hilum instead of cavo-atrial junction with its tip directed posteriorly (Figure 1\&2).

There was associated lung collapse due to hydro pneumothorax. Left sided chest drain was placed which drained $500 \mathrm{ml}$ hemorrhagic fluid. The diagnostic confusion regarding possible location of the catheter was resolved after CT angiography of chest which showed that the catheter had traversed the left internal jugular (LIJV) and brachiocephalic vein (BVC) into an anomalous vascular communication between left upper lobar pulmonary vein and brachiocephalic vein (Figure 3\&4). The catheter tip was lying in the distal part of left upper lobar pulmonary vein with linear hypodensity inside 
the vein suggesting possible thrombus. An incidental diagnosis of left PAPVC was established. Line was removed so was the chest tube once the lung fully expanded. Echo cardiograph did not demonstrate any other cardiac anomaly like atrial septal defect (ASD), left ventricular hypertrophy, pulmonary arterial hypertension.

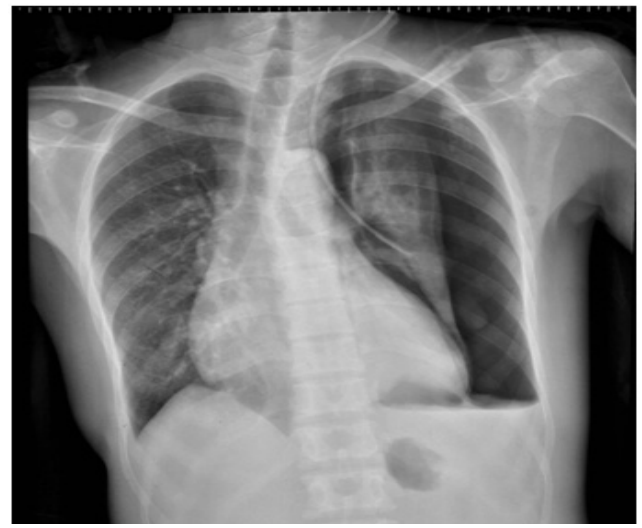

Figure 1: Chest radiograph showing left IJV catheter tip overlying left collapsed lung field with hydropneumothorax

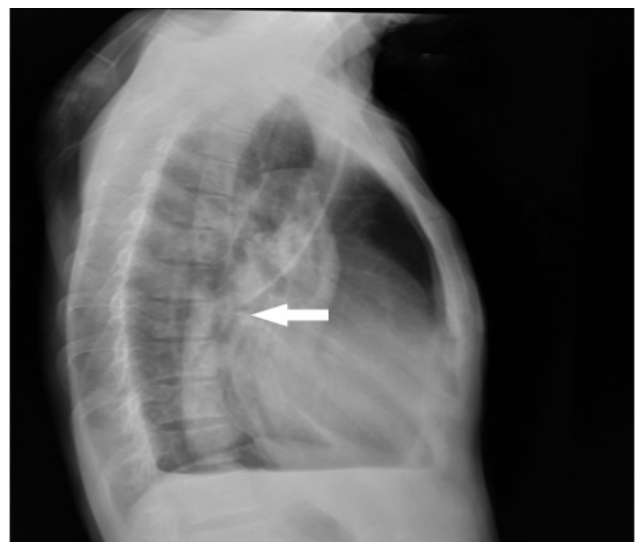

Figure 2: Lateral chest radiograph showing abnormal posterior course of the central venous catheter (white arrow)

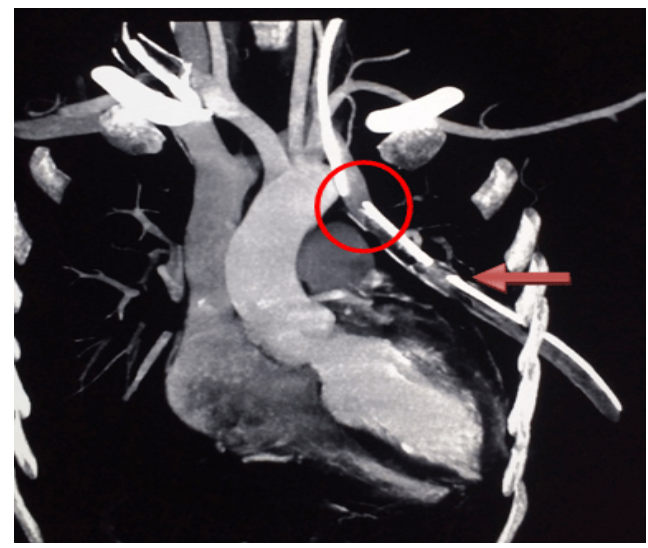

Figure 3: CT Angiography showing with left IJV catheter tip (red circle) in side left PAPVC with chest tube (red arrow) in situ

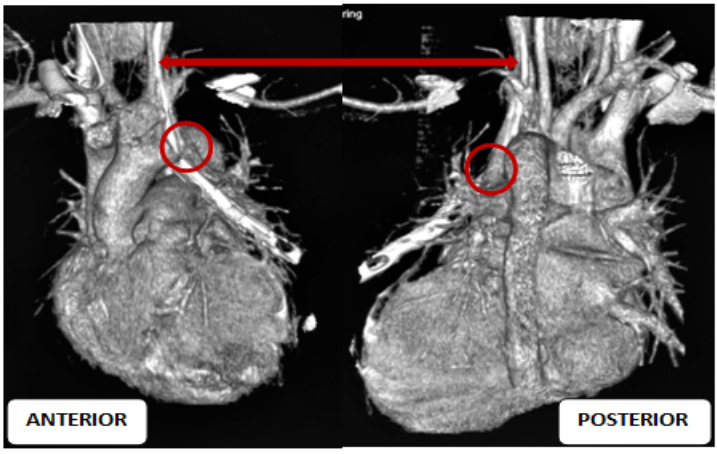

Figure 4: Volume rended images of heart and great showing tip of central line (red double head arrows) inside left PAPVC (red circles)

\section{Discussion}

PAPVC is a rare congenital anomaly causing left to right shunt akin to atrial septal defect [1]. Right PAPVCs drain into superior vena cava or right atrium while left ones drain into innominate vein. Majority remains asymptomatic and is detected incidentally as radiological surprise or at autopsy. Development of symptoms or complications depends on magnitude of left to right shunt [2]. When symptomatic they will produce symptoms and complications such as dyspnea, palpitation, chest pain, arrhythmia, recurrent chest infection or hemoptysis. Clinical evaluation in symptomatic patients may reveal evidence of right ventricular hypertrophy, elevated pulmonary pressures, and signs of right heart ischemia [1]. Asymptomatic PAPVCs do not warrant any intervention as in our case. In symptomatic cases goal of treatment is to redirect the blood from anomalous pulmonary vein to left atrium or to remove the pulmonary lobe bearing the anomalous connection.

Placement of central venous catheter (CVC) is one of the most common interventions performed in critical care setup in patients requiring invasive hemodynamic monitoring, inotropic support, parenteral nutrition, drug infusion and dialysis. These invasive lines are associated with significant number of complications even in the experienced hands. pneumothorax, bleeding inside pleural cavity or mediastinum, hematoma formation, infections are seen in up to one third of cases [3]. Incidence of accidental arterial catheterization is $0.1-1 \%$ [4]. Malpositioning of CVC in wrong vein, wrong position or wrong direction are seen in up to $7 \%$ cases [3]. If overlooked they can result in false central venous pressure reading, unsatisfactory infusion rate, and vessel erosion or even rupture, venous thrombosis and retrograde infusion into cranium. Venous malpositioning is more frequent in left sided lines and hence usually avoided [5,6]. Our patient already had a dialysis access placed in right IJV so left sided IJV was used for access. Rarely presence of congenital venous anomalies like left sided SVC, dominant supreme intercostal vein, PAPVC can result inmalpositioning as in our case. Puncture related pneumothorax, haemothorax, venous injury, and accidental arterial puncture and subsequent catheterization can cause significant morbidity and even death. Most of these technical complications are avoided if placement is done under image guidance [4]. But image guided placement may not give total immunity against

Citation: Shibojit Talukder, et.al (2017) Accidental Catheterization of Left Partial Anomalous Pulmonary Venous Connection (PAPVC) A Rare Instance of Central Venous Access Malpositioning. J Cardiovascular Thoracic Surgery. 2(3):1-3. DOI: 10.15226/2573- 
tip malpositioning which is influenced heavily by body habitus, congenital or acquired anatomical variation in neck and thoracic anatomy and puncture technique [3].

Accidental catheterization of pulmonary artery during subclavian venous access placement has been reported but there is no such report on venous counterpart [7]. Since the PAPVC in our patient was in linear alignment with left IJV the catheter passed via it into left upper pulmonary vein. Presence haemo-pneumothorax raised suspicion of major vascular injury along with pleural breach. Retrospectively these together can be explained by injury to the pulmonary vein and lung parenchyma due to overzealous advancement of the guide wire and catheter in an attempt by the operator to position the tip of the line at cavo-atrial junction which is sometimes difficult to achieve in left sided CVC insertion. Catastrophic bleeding probably did not occur in our patient due do thrombus formation in the pulmonary vein.

Clinically malposition should be suspected when there is poor backflow in ports, resistance to infusion, infusion related chest pain, arm pain, head ache, gurgling sound in ear [3]. However, low resistance to saline flush or free backflow do not always rule out malpositioning. So post insertion postero-anterior view chest radiograph is mandatory to check tip position and identifying procedure related complications. Whenever there is clinical and radiological suspicion of malpositioning further investigation is warranted either in form of a lateral view skiagram or cross sectional imaging. Dynamic radiograph while injecting small volume contras into CVC port can also help in delineating venous anatomy and confirm tip location $[7,8]$.

A malpositioned catheter should be repositioned, replaced or removed depending on scenario [3]. Some malpositions are amenable to fluoroscopy guided repositioning over guide wire while some malpositions are compatible with clinical use in patients with difficult venous access. However catheter inside artery or pulmonary vasculature should be removed as soon as possible. If removal carries risk of bleeding from puncture point which cannot be compressed externally endovascular or surgical procedure may have to be resorted to [3].

\section{Declaration}

\section{Ethical Approval}

This study involved human subject participation. All the invasive procedures done to the patient namely surgery, central line insertion and chest tube placement were carried out after obtaining valid informed consent from the patients as per institutional protocol. All the treatments provided were also in accordance with the institutional practice. All attempts have been made to prevent disclosure of identity and personal information of the patient. All procedures performed in studies involving human participants were in accordance with the ethical standards of the institutional and/or national research committee and with the 1964 helsinki declaration and its later amendments or comparable ethical standards.

\section{Reference}

1. Basalus MW, Said SA, Stassen CM, Fast JH. Clinical and diagnostic features of partially anomalous pulmonary venous connection in an adult female patient: a case report and review of the literature. Neth Heart J. 2011;19(5):256-258. doi: 10.1007/s12471-011-0101-9

2. Darryl S. Weiman, Ken Lee, James M. Levett, Robert L. Replogle. Partial anomalous pulmonary venous return: a ten-year experience. Tex Heart Inst J. 1985;12(3):239-243

3. Roldan CJ, Paniagua L. Central venous catheter intravascular malpositioning: causes, prevention, diagnosis, and correction. West J Emerg Med. 2015;16(5):658-664. doi: 10.5811/westjem.2015.7.26248

4. Bowdle A. Vascular complications of central venous catheter placement: evidence-based methods for prevention and treatment. J Cardiothorac Vasc Anesth. 2014;28(2):358-368. doi: 10.1053/j. jvca.2013.02.027

5. Schummer W, Schummer C, Rose N, Niesen WD, Sakka SG. Mechanical complications and malpositions of central venous cannulations by experienced operators. Intensive Care Med. 2007;33(6):1055-1059. doi: 10.1007/s00134-007-0560-z

6. McGee DC, Gould MK. Preventing complications of central venous catheterization. N Engl J Med. 2003;348(12):1123-1133. doi: 10.1056/ NEJMra011883

7. Jérôme Moriceau, Vincent Compère, Marc Bigo, Bertrand Dureuil. Accidental puncture of the pulmonary artery during a subclavian central venous catheterization. Case reports in critical care. 2012;2012:3. doi: 10.1155/2012/160847

8. Funaki B. Diagnostic and interventional radiology in central venous access. Seminars in Roentgenology. 2002;37(4):343-353. doi: 10.1016/ S0037-198X(02)80010-4 